\title{
A SIMPLE DESCRIPTION OF THE STRUCTURE OF IONIC SOLUTIONS AT ELECTRIFIED INTERFACES
}

\author{
J.StAFIEJ $^{(1)}$, A.EKoKA ${ }^{(2)}$, Z.BorKowskA ${ }^{(1)}$, J.P.BADIALI ${ }^{(2)}$ \\ (1) Institute of Physical Chemistry \\ of the Polish Academy of Sciences \\ ul. Kasprzaka 44/52, 01-224 Warsaw, Poland \\ ${ }^{(2)}$ Structure et Réactivité des Systèmes Interfaciaux \\ Université $P$. et M. Curie \\ Bât. F, BP 52, 4. Place Jussieu, 75230 Paris Cedex 05, France
}

Received January 17, 1997

\begin{abstract}
In this paper a field theoretical approach is used in the description of the properties of ionic solutions near a charged wall. The differences with the Gouy-Chapman theory are emphasized both near the point of zero charge and for high values of the charge. At the point of zero charge we are getting a maximum instead of a minimum and at high charge densities we observe a saturation in the capacitance values instead of a continuous increase. The results are very sensitive to the asymmetry of ions which is expected in real systems. Our approach seems to be a good starting point for investigating various phenomena at charged interfaces and in the bulk of ionic solutions.
\end{abstract}

\section{Introduction}

The main goal of standard statistical mechanics is to describe the macroscopic properties of systems in terms of quantities which are defined at the microscopic level - the intermolecular potentials. In order to do that several starting points, in principle equivalent, can be used. For instance, we can start from the grand canonical ensemble and derive from it the so-called integral equations. They have been very successful in describing the properties of hard sphere fluids or the structure of ionic solutions, see, for example, [1]. Another choice consists in starting from the density functional theory which asserts that the equilibrium distribution of particles corresponds to the minimum of an appropriate free energy functional. This approach has been extensively used to describe interfacial properties including the wetting phenomena [2]. For the given system defined at a microscopic level, each description requires some assumptions that can be verified by comparison with numerical simulations (Monte-Carlo or molecular dynamics).

When we deal with complicated systems it may turn out too ambitious to try to have a description in terms of intermolecular pair potentials. This is particularly true when we attempt to investigate the structure of membranes or micellar solutions. In these cases we have to consider a mixture of several entities for which the pair potential is not very well known and, moreover, we do not know whether a description in terms of pair potential is accurate enough. As another example we may consider the ideally polarizable electrode which is the simplest metal-solution interface, since in the stationary regime no electrical current crosses the interface. To describe real experiments we have to know the interfacial electronic structure of the

(c) J.Stafiej, A.Ekoka, Z.Borkowska, J.P.Badiali, 1997

ISSN 0452-9910. Condensed Matter Physics 1997 No 12 (51-62) 
electrode, the perturbation of it induced by the solution side, the adsorption of molecules or ions onto the metallic interface and the details of the ionmolecule interaction in order to describe the solvation of ions. In addition, we know that interfacial properties are nonlinear functions of the interfacial potential or the charge on the metal. Of course, it is possible to treat the above problems separately and to perform a comparison with simulations for the isolated problem independently of the other problems. Nevertheless, at the end we will have to restore the coupling between several parts investigated separately. From the late 50-ies starting with the work of Watt and Mott-Tobin, the so-called molecular models were developed in which the overall interface has been studied [3-4]. These models contributed to a substantial progress in understanding the electrified interface. However, these models include several parameters and, in general, different models can describe the same experiments equally well. As a consequence, it is difficult to assess the validity of a particular microscopic model from experimental data. Evidently, more direct measurements of interfacial properties are needed to validate these models.

In recent papers another approach has been proposed [5-8]. It is less ambitious than those discussed above. The general philosophy behind it is the following. Since the description of a specific experiment is a difficult task it should be more simple to consider a collection of many experiments, to observe gross features common to some classes of the experiments and then to investigate if we can reproduce these general features by using very general physics. In other words, can we predict different kinds of behavior for the interface just by considering some well-known ingredients in physics? Behind this approach another question emerges - what kind of information we can really get from the experiments? In this paper we will work in this direction in order to study some aspects of the ionic adsorption at a charged interface.

When we investigate the properties of charged interfaces, the GouyChapman theory remains up to now an indispensable tool in the interpretation of experimental data. The more sophisticated and up-to-date theories (MSA, HNC, ...) [9] have never acquired its significance. This is due to the fact the Gouy-Chapman theory (or equivalently the Poisson-Boltzmann theory) is based on simple and intuitive arguments. However, the GouyChapman theory itself is not able to account for the experimental data especially in concentrated electrolyte solutions. For this reason it was completed by Grahame [10] with the notion of the compact or inner layer first introduced by Stern [11]. In what follows we will try to stay as close as possible to the spirit in which the Gouy-Chapman theory was initially derived. As we shall see, the approximations introduced in our extension of the Gouy-Chapman theory are simple and have a well-defined physical meaning. They are also sufficient to obtain a lot of different behaviors for the electrical properties, of the interface. Some of these properties not accounted for by the Gouy-Chapman theory, were previously associated with the specific ionic adsorption.

This paper is arranged as follows. In section 2 we briefly summarize the field theoretical approach which we use and discuss the meaning of the coupling constants which appear in the Hamiltonian. In section 3 we describe some analytical results concerning the differential capacitance of the interface. They clearly show deviations from the Gouy-Chapman theory. In section 4 we analyze some predictions of our model. Conclusions and perspectives are presented in section 5 . 


\section{Theoretical approach}

\subsection{The formalism}

As in the standard Gouy-Chapman theory our main goal is to describe the distribution of ions in a dielectric continuum near a charged wall. We consider a planar interface in which $z$ is a direction normal to the wall. We assume that the partition function $Z$ of the interface can be written as a functional integral according to

$$
Z=Z^{i r r} \int D \rho^{+}(\mathbf{r}) \mathbf{D} \rho^{-}(\mathbf{r}) \exp \left\{-\beta \mathbf{H}^{e f f}\left[\rho^{+}(\mathbf{r}), \rho^{-}(\mathbf{r})\right]\right\},
$$

where $\rho^{+}(\mathbf{r})$ and $\rho^{-}(\mathbf{r})$ are two fields which represent the concentrations of cations and anions at the point $\mathbf{r}$ of the space and $\beta=1 / k_{\mathrm{B}} T$. The free energy of the interface will be given by the following standard expression:

$$
F=F^{i r r}-k_{\mathrm{B}} T \ln \left(\int \mathcal{D} \rho^{+}(\mathbf{r}) \mathcal{D} \rho^{-}(\mathbf{r}) \exp \left\{-\beta \mathcal{H}^{e f f}\left[\rho^{+}(\mathbf{r}), \rho^{-}(\mathbf{r})\right]\right\}\right)
$$

in which $F^{i r r}$ contains a charge independent part of the free energy. We expect that the integral which appears in (2.2) contains the main features of the charge dependence while the Hamiltonian $H^{\text {eff }}\left[\rho^{+}(\mathbf{r}), \rho^{-}(\mathbf{r})\right]$ retains a very transparent physical interpretation. We have no strict proof that this is possible but we know that the equivalence between a field theoretic approach and the standard statistical mechanics has been already established in given conditions from which the ionic screening has been derived. Moreover, a contact between a field theoretic approach and the standard liquid state theory has been already presented. As we shall see below, we may also expect the validity of such an approach because it allows one to derive some standard results as the Non-Linear-Gouy-Chapman (NLGC) theory [6-7] in a simple way. In particular, we may expect that such an approach is justified when one length is dominating compared to the other ones; this is the case at very low ionic concentrations when the Debye length is large compared to the size of the ionic core. To our knowledge, a similar approach using a Landau type Hamiltonian has been already proposed for bulk ionic solutions by Nabutovskii et al [12] and after that by Hoye and Stell [13]. But in these two cases the authors focus on the critical regime in the bulk phase.

The meaning of the integral in (2.2) is the following. We select arbitrary values for the fields $\rho^{+}(\mathbf{r})$ and $\rho^{-}(\mathbf{r})$ at each point of a cubic grid $\left(\mathbf{r}_{i}\right)$ in the interface which formally extends to the solution halfspace away from the wall. In general, the Hamiltonian $\beta \mathcal{H}^{\text {eff }}\left[\rho^{+}\left(\mathbf{r}_{i}\right), \rho^{-}\left(\mathbf{r}_{i}\right)\right]$ may be a complicated function of a set of values of the both fields at all the points of the grid. The function describes the coupling of the fields with themselves locally, at a given point, as well as non locally at two or more different points. In the functional integral we integrate the Boltzmann factor varying the field values at each point of the grid independently and then proceeding to the continuous limit.

We assume [7] that $\beta \mathcal{H}^{e f f}\left[\rho^{+}(\mathbf{r}), \rho^{-}(\mathbf{r})\right]$ can be written as :

$$
\beta \mathcal{H}^{e f f}\left[\rho^{+}(\mathbf{r}), \rho^{-}(\mathbf{r})\right]=\beta \mathcal{H}\left[\rho^{+}(\mathbf{r}), \rho^{-}(\mathbf{r})\right]-\ln \Omega\left[\rho^{+}(\mathbf{r}), \rho^{-}(\mathbf{r})\right],
$$

where $\Omega\left[\rho^{+}(\mathbf{r}), \rho^{-}(\mathbf{r})\right]$ is the number of states corresponding to the fields 
$\rho^{+}(\mathbf{r})$ and $\rho^{-}(\mathbf{r})$. It is given by [7]:

$$
\ln \Omega\left[\rho^{+}(\mathbf{r}), \rho^{-}(\mathbf{r})\right]=\int\left\{\rho^{+}(\mathbf{r})\left[-\ln \frac{\rho^{+}(\mathbf{r})}{\rho}+1\right]+\rho^{-}(\mathbf{r})\left[-\ln \frac{\rho^{-}(\mathbf{r})}{\rho}+1\right]\right\} \mathrm{d} \mathbf{r}
$$

where $\rho$ plays a role similar to the de Broglie wave length in the usual statistical mechanics. As we shall see later, it disappears in the mean field approximation. tional:

The Hamiltonian $\mathcal{H}\left[\rho^{+}(\mathbf{r}), \rho^{-}(\mathbf{r})\right]$ contains the coulombic energy func-

$$
\beta \mathcal{H}_{q}\left[\rho^{+}(\mathbf{r}), \rho^{-}(\mathbf{r})\right]=\frac{1}{2} \frac{\beta e^{2}}{4 \pi \epsilon} \int \mathrm{d} \mathbf{r} \mathrm{d} \mathbf{r}^{\prime} \frac{[q 0(\mathbf{r})+q(\mathbf{r})]\left[q 0\left(\mathbf{r}^{\prime}\right)+q\left(\mathbf{r}^{\prime}\right)\right]}{\left|\mathbf{r}-\mathbf{r}^{\prime}\right|},
$$

where $e$ is elementary charge, $\epsilon$ is dielectric permitivity of the dielectric continuum, $q(\mathbf{r})=\rho^{+}(\mathbf{r})-\rho^{-}(\mathbf{r}), q 0(\mathbf{r})=e \sigma \delta(z)$ is charge distribution behind the wall and $e \sigma$ is surface charge density. We have placed the wall at $z=0$. In addition to $\beta \mathcal{H}_{q}\left[\rho^{+}(\mathbf{r}), \rho^{-}(\mathbf{r})\right]$ we introduce $\beta \mathcal{H}_{2}\left[\rho^{+}(\mathbf{r}), \rho^{-}(\mathbf{r})\right]$ which takes into account the fluctuations of $\rho^{+}(\mathbf{r})$ and $\rho^{-}(\mathbf{r})$. It is given by:

$\left.\beta \mathcal{H}_{2}\left[\rho^{+}(\mathbf{r}), \rho^{-}(\mathbf{r})\right]=\frac{1}{2 \rho_{b}} \int\left\{a_{++}\left[\rho^{+}(\mathbf{r})\right]^{2}+2 a_{+-} \rho^{+}(\mathbf{r}) \rho^{-}(\mathbf{r})+a_{--}\left[\rho^{-}(\mathbf{r})\right]^{2}\right]\right\} \mathrm{d} \mathbf{r}$,

where $\rho_{b}$ is the overall bulk ionic concentration i.e. twice the electrolyte concentration. The quantities $a_{++}, a_{+-}$, and $a_{--}$are dimensionless parameters describing the correlations between ions [7].

The relevant part of the free energy corresponding to $\beta \mathcal{H}^{e f f}\left[\rho^{+}(\mathbf{r}), \rho^{-}(\mathbf{r})\right]$ can be calculated exactly using (2.2). In this paper we only focus on the mean field determination of this free energy. In this case the average profiles are obtained by solving two equations:

$$
\begin{aligned}
& \frac{\delta}{\delta \rho^{+}(\mathbf{r})}\left\{\beta \mathcal{H}^{e f f}\left[\rho^{+}(\mathbf{r}), \rho^{-}(\mathbf{r})\right]-\mu_{+} \int \rho^{+}(\mathbf{r}) \mathrm{d} \mathbf{r}\right\}=0, \\
& \frac{\delta}{\delta \rho^{-}(\mathbf{r})}\left\{\beta \mathcal{H}^{e f f}\left[\rho^{+}(\mathbf{r}), \rho^{-}(\mathbf{r})\right]-\mu_{-} \int \rho^{-}(\mathbf{r}) \mathrm{d} \mathbf{r}\right\}=0,
\end{aligned}
$$

which express the necessary condition for the free energy to have a minimum with respect to any variation of the profiles. In these equations the symbols $\delta / \delta \rho^{+}(\mathbf{r})$ and $\delta / \delta \rho^{-}(\mathbf{r})$ denote functional differentiation. Due to the symmetry of the wall the average profiles are z-dependent only. In equations (2.7) and (2.8) two Lagrange multipliers, $\mu_{+}$and $\mu_{-}$, are introduced to account for the fact that the total number of cations and anions is fixed during the minimization. They can be eliminated by subtracting the bulk contribution from each equation. The conditions for the minimum of the free energy can be transformed to the form:

$$
\begin{gathered}
{\left[\frac{2}{g^{+}(z)}+a_{++}\right] \frac{\mathrm{d} g^{+}(z)}{\mathrm{d} z}+a_{+-} \frac{\mathrm{d} g^{-}(z)}{\mathrm{d} z}=s(z),} \\
a_{+-} \frac{\mathrm{d} g^{+}(z)}{\mathrm{d} z}+\left[\frac{2}{g^{-}(z)}+a_{--}\right] \frac{\mathrm{d} g^{-}(z)}{\mathrm{d} z}=-s(z),
\end{gathered}
$$




$$
\frac{d s(z)}{d z}=g^{+}(z)-g^{-}(z),
$$

where we have expressed $z$ in Debye length unit (i.e. $z$ is the product of the distance to the wall with the reciprocal Debye length $K_{\mathrm{D}}=e\left(\rho_{b} \beta / \epsilon\right)^{\frac{1}{2}}$. We have also introduced $g^{+}(z)=2 \rho^{+}(z) / \rho_{b}$ and $g^{-}(z)=2 \rho^{-}(z) / \rho_{b}$. In the above equations $s(z)$ is defined as

$$
s(z)=\int_{\infty}^{z}\left[g^{+}\left(z^{\prime}\right)-g^{-}\left(z^{\prime}\right)\right] \mathrm{d} z^{\prime}
$$

and $s(z)$ is proportional to the electric field $E(z)$. At the point $z$ we have $E(z)=s(z) K_{\mathrm{D}} / 2 \beta \epsilon$. From now on we refer to $s(z)$ as either electric field or charge density having in mind that these are equivalent in the sense that they differ by the conversion constant only. It is readily seen that equation (2.11) is just the Poisson equation expressed in reduced variables. In addition to the differential equation system (2.9)-(2.11) we have two boundary conditions for $s(z)$ :

$$
\begin{gathered}
\left.s(z 0)\right|_{z=0}=\frac{2 \beta e}{\epsilon K_{\mathrm{D}}} \sigma, \\
s(z) \rightarrow 0 \text { when } z \rightarrow \infty ;
\end{gathered}
$$

and this implies for the profiles

$$
g^{+}(z) \rightarrow 1 \text { and } g^{-}(z) \rightarrow 1 \text { when } z \rightarrow \infty .
$$

Due to the fact that (2.7) and (2.8) are not a sufficient condition for the minimum of free energy we have to complete it with the condition that the second derivative of free energy is a positive definite bilinear form of the field. This leads to the following inequalities:

$$
\begin{gathered}
\frac{2}{g^{+}(z)}+a_{++}>0 \\
\frac{2}{g^{-}(z)}+a_{--}>0, \\
\left(\frac{2}{g^{+}(z)}+a_{++}\right)\left(\frac{2}{g^{-}(z)}+a_{--}\right)-a_{+-}^{2}>0,
\end{gathered}
$$

which must be verified at any point $z$. It is easy to see that in the case where $a_{++}=a_{+-}=a_{--}=0$, the ionic distributions $g^{+}(z)$ and $g^{-}(z)$ correspond to the NLGC theory. We can see that the coefficient $\rho$ which we have introduced in (2.4) has been eliminated by subtracting the bulk properties.

\subsection{The meaning of $a_{++}, a_{--}$and $a_{+-}$}

The profiles $g^{+}(z)$ and $g^{-}(z)$ resulting from $(2.9-2.11)$ depend on the three parameters $a_{++}, a_{--}$and $a_{+-}$only. These parameters account for the fact that a realistic Hamiltonian $\beta \mathcal{H}^{e f f}\left[\rho^{+}(\mathbf{r}), \rho^{-}(\mathbf{r})\right]$ should contain more information on specific interionic interaction than a simple combination 
of the ideal entropy term $\ln \Omega\left[\rho^{+}(\mathbf{r}), \rho^{-}(\mathbf{r})\right]$ and the electrostatic energy $\beta \mathcal{H}_{q}\left[\rho^{+}(\mathbf{r})-\rho^{-}(\mathbf{r})\right]$. They lead to a deviation from the NLGC theory. In terms of the field theory they appear as coupling constants. Let us note that $\beta \mathcal{H}_{2}\left[\rho^{+}(\mathbf{r}), \rho^{-}(\mathbf{r})\right]$ can be rewritten according to

$$
\beta \mathcal{H}_{2}\left[\rho^{+}(\mathbf{r}), \rho^{-}(\mathbf{r})\right]=\frac{1}{2 \rho_{b}} \sum_{i, j=+.-} \int a_{i j} \rho^{i}(\mathbf{r}) \delta\left(\mathbf{r}-\mathbf{r}^{\prime}\right) \rho^{j}\left(\mathbf{r}^{\prime}\right) \mathrm{d} \mathbf{r} \mathrm{d} \mathbf{r}^{\prime}
$$

Considering the above form we may say that we are investigating a system in which there is a dominating length. For dilute solutions it is the Debye length. The other lengths are small compared to it and the spatial dependence of short range interactions or correlations can be conveniently represented by the Dirac function $\delta(\mathbf{r})$. This does not imply that we are treating point particles. Such an approach has been already used in order to describe the long range properties of polar fluids [14]. Now we see that the coefficients $a_{i j}$ determine the magnitude of the short range correlation in the system.

It is noteworthy that the coefficients $a_{i j}$ are specific of a given ionic solution. Accordingly, they also determine the properties of the bulk solution. If we cancel the external field i.e. the charged wall which originates the inhomogeneity in the system, then $\beta \mathcal{H}^{e f f}\left[\rho^{+}(\mathbf{r}), \rho^{-}(\mathbf{r})\right]$ can be used for describing the structure of the ionic solution. Clearly, the coefficients $a_{i j}$ now determine the deviation from the Debye-Hückel theory. It is easy to show that they are related to the non- coulombic part of the activity coefficient calculated in the mean field theory. This might represent one route from which they can be related to the microscopic description of the system.

If we want to recover the Gouy-Chapman theory which is exact at infinitely low concentrations, we can see from (2.8) that $a_{++}, a_{--}$and $a_{+-}$ must be concentration dependent. Since the quadratic fluctuations are not primarily related to the electrostatic effects for which a non-analytic behavior is expected, the simplest choice consists in assuming that these coefficients are proportional to the ionic concentration. We will write $a_{i j}=\rho_{b} \alpha_{i j}$ where we may assume that $\alpha_{i j}$ is concentration independent, at least, at very low concentrations. Of course, the magnitude of the coefficients $\alpha_{i j}$ is also determined by the ion-solvent interaction. The difference $a_{++}-a_{--}$ (or $\alpha_{++}-\alpha_{--}$) gives an indication of the asymmetry between ions.

\section{Analytical results}

The expression for the differential capacitance $C$ has been already established [8]. It reads:

$$
C=e \frac{\mathrm{d} \sigma}{\mathrm{d} V}=\epsilon K_{\mathrm{D}}\left(-\frac{g_{0}^{+}-g_{0}^{-}}{s_{0}}\right),
$$

where $s_{0}, g_{0}^{+}$and $g_{0}^{-}$are the values of the profiles $s(z), g^{+}(z)$ and $g^{-}(z)$ for $z=0$. Let us recall that (2.13) yields $s_{0}=\frac{2 \beta e}{\epsilon K_{\mathrm{D}}} \sigma$. All the deviations of the capacitance from the value of the Gouy-Chapman capacitance at the point of zero charge, $\epsilon K_{\mathrm{D}}$, are determined by the ratio of dimensionless quantities $\left(g_{0}^{+}-g_{0}^{-}\right) / s_{0}$. Of course, the non-linear Gouy-Chapman theory also verifies (3.1). 


\subsection{Capacitance near the point of zero charge}

The capacitance at the zero charge density on the wall, $C_{0}$, is given by

$$
C_{0}=\epsilon K_{\mathrm{D}} \sqrt{\frac{4+a_{++}+a_{--}+2 a_{+-}}{\left(2+a_{++}\right)\left(2+a_{--}\right)-a_{+-}^{2}}}
$$

and its first derivative with respect to $\sigma, C_{0}^{\prime}$, is given by

$$
C_{0}^{\prime}=2 \beta e \epsilon K_{\mathrm{D}}\left(a_{++}-a_{--}\right) \frac{\left(2+a_{++}\right)\left(2+a_{--}\right)-3 a_{+-}\left(4+a_{++}+a_{--}\right)}{3 C_{0}^{2}\left[\left(2+a_{++}\right)\left(2+a_{--}\right)-a_{+-}^{2}\right]^{2}} .
$$

If we consider that the concentration approaches zero, we have the GouyChapman result $C_{0}=\epsilon K_{\mathrm{D}}$. For low concentrations the relation (3.2) predicts a deviation from the Gouy-Chapman theory. The capacitance is given by $C_{0}=\epsilon K_{\mathrm{D}}\left(1+\rho_{b} A\right)$ where $A$ is a combination of the coefficients $\alpha_{i j}$. For these concentrations, if the ions are not symmetrical i.e. if $\left(a_{++}-a_{--}\right)=\rho_{b}\left(\alpha_{++}-\alpha_{--}\right)$does not vanish, we can see from (3.3) that $C_{0}^{\prime}$ is non-zero. Then $C_{0}$ is not the minimum of the capacitance. Conversely, as a consequence, the minimum of the $C(\sigma)$ curve does not correspond to the zero charge value and the slope at the pzc is equal to $K_{\mathrm{D}}\left(\alpha_{++}-\alpha_{--}\right) / 6$ and depends on the square root of the ionic concentration.

If we are in more concentrated solutions, $C_{0}$ and $C_{0}^{\prime}$ are still given by (3.2) and (3.3), but the extremum on the $C(\sigma)$ curve observed for symmetric ions at the zero charge on the wall can be a maximum, as well as a minimum. At the maximum it is rather difficult to change the potential changing the charge density in contrast to the case when we have a minimum. For asymmetrical ions we may observe a given finite slope at zero charge instead of an extremum. These results are illustrated in figures 1 and 2 . We will discuss the two figures in more detail later.

\subsection{Capacitance at high values of the charge density}

It is also possible to calculate the interfacial capacitance in the regime: $\sigma \rightarrow \pm \infty$. If $a_{--}$and $a_{++}$do not vanish, then selecting the dominant terms in (2.9)-(2.11) we obtain:

$$
\begin{aligned}
& C \rightarrow \frac{\epsilon K_{\mathrm{D}}}{\sqrt{a_{--}}}=\sqrt{\frac{\beta \epsilon e^{2}}{\alpha_{--}}} \text {for } \sigma \rightarrow+\infty, \\
& C \rightarrow \frac{\epsilon K_{\mathrm{D}}}{\sqrt{a_{++}}}=\sqrt{\frac{\beta \epsilon e^{2}}{\alpha_{++}}} \text {for } \sigma \rightarrow-\infty .
\end{aligned}
$$

The above limits are finite for $a_{++}>0$ and $a_{--}>0$. Then the model leads to a saturation for the values of the capacitance. When $\sigma \rightarrow+\infty$ the structure of the interface is mainly determined by the anions, as expected. Moreover, if $\alpha_{--}$is concentration independent, then the asymptotic value of the capacitance is also concentration independent. A change $\delta \sigma$ in the charge density produces the change of the interfacial potential $V$ by $\delta V=$ $\delta \sigma \sqrt{\alpha_{--} / \beta \epsilon e^{2}}$. We see that the larger is $a_{--}$, the larger will be $\delta V$, and the smaller the limiting capacitance.

The relations (3.4) and (3.5) also show that the interfacial free energy behaves like $\sigma^{2}$, which is more realistic than the $\sigma^{3}$ asymptotic dependence 
observed in the Gouy-Chapman theory. If we compare (3.1) with (3.4) and (3.5), we can see that $g_{0}^{+}-g_{0}^{-}$behaves like $\sigma$, while in the non-linear Gouy-Chapman theory it behaves like $\sigma^{2}$. Here, on the contrary, the GouyChapman theory is more accurate.

In order to conclude this part we see that we have a very simple model including short range correlations between ions. They are represented by quadratic terms in the Hamiltonian which is the simplest correction that we can imagine. In this respect we can say that the functional form of $\beta \mathcal{H}^{e f f}\left[\rho^{+}(z), \rho_{-}(z)\right]$ is universal. All the specificities of the problem are localized in the three coupling constants $a_{i j}, i, j=+$ or - . With the same Hamiltonian $\left.\beta \mathcal{H}^{\text {eff }}\left[\rho^{+}(z)\right), \rho_{-}(z)\right]$ we are able to produce different kinds of behavior just by changing the magnitude of parameters $a_{i j}$. At very low ionic concentrations our model is identical with the NLGC theory. From now on we shall refer to it as GGCM (Generalized Gouy - Chapman Model)

\section{Ionic adsorption}

Specific ionic adsorption is assumed to exist whenever the surface excess of a given ion is greater than that predicted by the Gouy-Chapman theory [15]. So the validity of this theory is crucial in the investigation of ionic adsorption at electrodes. In order to obtain the ionic profiles and the capacitances as a function of charge density we have to solve equations (2.9-2.11). This can be done by the numerical procedure presented in [8]. Some examples of the results are given in figures 1 and 2 where we can see the influence of the parameters $a_{i j}$. In all cases we accept the value of the dielectric constant to be 78.0 as for water and the temperature of $298.15 \mathrm{~K}$.

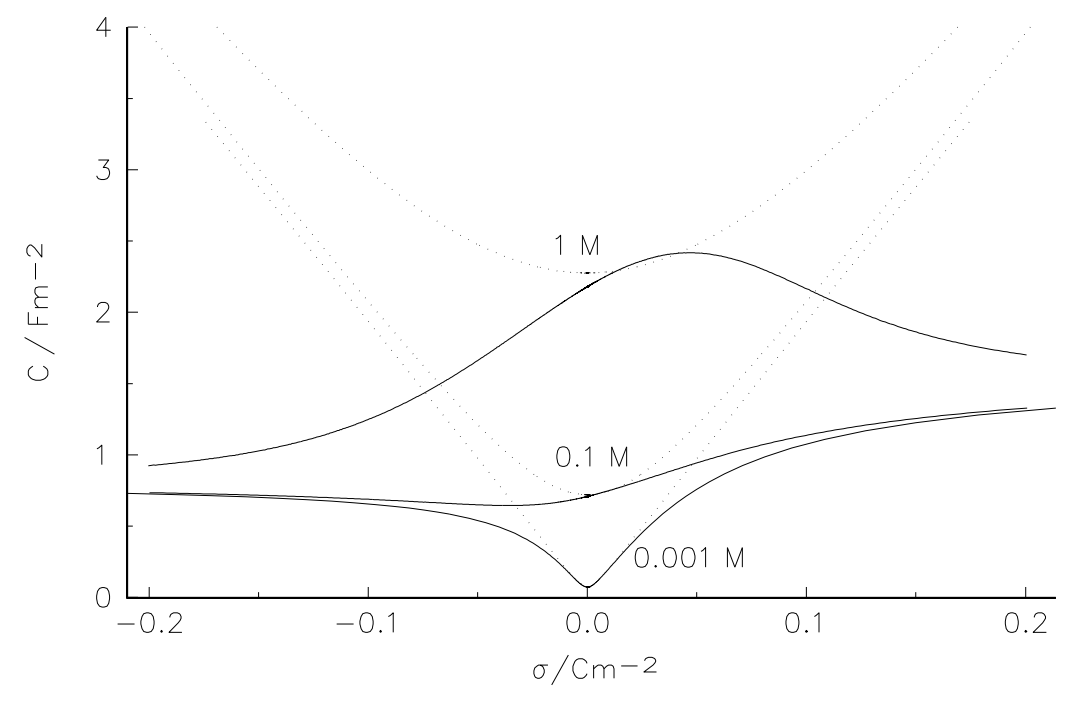

Figure 1. The symmetric case. Capacitance $\mathrm{C}$ as a function of the charge density $\sigma$ on the wall calculated according to the NLGC theory $\left(C_{G C}\right.$, dotted lines) and our model $\left(C_{G G C}\right.$, full lines) with the following parameter set: $a_{++}=a_{+-}=a_{--}=2 c_{\text {salt }} / 1 M$. The concentrations are given on the figure. 


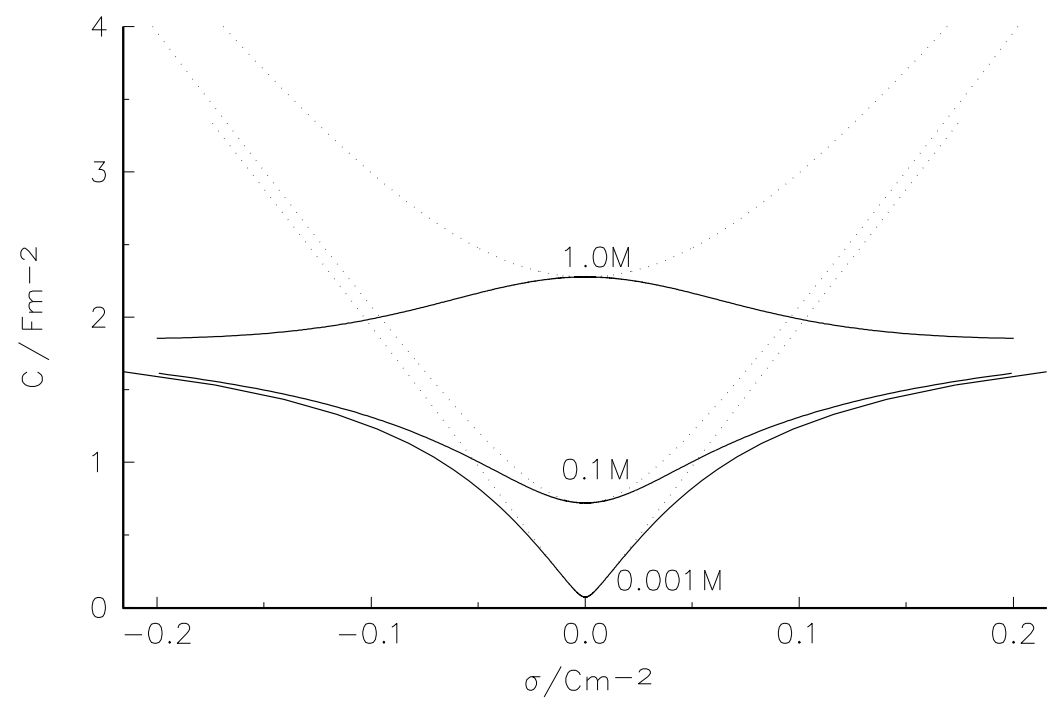

Figure 2. The asymmetric case. Capacitance $\mathrm{C}$ as a function of the charge density $\sigma$ on the wall calculated according to the NLGC theory $\left(C_{G C}\right.$, dotted lines) and our model $\left(C_{G G C}\right.$, full lines) with the following parameter set: $a_{++}=8 c_{\text {salt }} / 1 M, a_{--}=2 c_{\text {salt }} / 1 M$ and $a_{+-}=4 c_{\text {salt }} / 1 M$. The concentrations are given in the figure.

Figure 1 corresponds to a symmetrical case (all the parameters $a_{i j}$ are equal). We take the value $a_{++}=a_{+-}=a_{+-}=2 c_{\text {salt }} / c_{\text {ref }}$ where $c_{\text {salt }}$ is the salt molar concentration and we decide to take $c_{\text {ref }}=1 M$. It is noteworthy that for these values of the parameters we accept fluctuations in the free energy having $2 k_{\mathrm{B}} T$ as the order of magnitude at $1 M$ solution. The values of the capacitance which result from our model, $C_{G G C}$, are identical with those of the NLGC theory, $C_{G C}$, at the zero charge only. The most striking differences between the two models are visible at high concentrations and at high charge density limits. At high concentrations we observe a maximum of $C_{G G C}$ instead of the minimum of $C_{G C}$. At high charge densities $C_{G G C}$ goes to a constant value according to (3.4) and (3.5) when $C_{G C}$ increases indefinitely. In figure 2 an asymmetric case is presented. The parameter values are: $a_{++}=8 c_{\text {salt }} / c_{\text {ref }}, a_{--}=2 c_{\text {salt }} / c_{\text {ref }}$ and $a_{+-}=4 c_{\text {salt }} / c_{\text {ref }}$. For the unchardged wall $C_{G G C}$ and $C_{G C}$ coincide only for low concentrations. For $0.1 M$ solution the minimum of $C_{G G C}$ is shifted towards the negative charge densities as compared with $C_{G C}$ while for $1 M$ solution $C_{G G C}$ exhibits a maximum at the positive charge densities.

As already mentioned, the Gouy-Chapman theory plays a major role in the analysis of the experimental data. The Gouy-Chapman capacitance $C_{G C}$ is related to the Debye length and does not contain any specific properties, in particular, - no interfacial properties. Thus, to get some interfacial data we have to compare $C_{G C}$ and the experimental capacitance $C_{\text {exp }}$. It is generally assumed that the overall interface can be described by two capacitances in a series model and we write

$$
\frac{1}{C_{\exp }}=\frac{1}{C_{i}}+\frac{1}{C_{G C}}
$$


where $C_{i}$ represents the so-called inner layer capacitance. For electrochemists it is $C_{i}$ that contains the information about the interface (the typical order of magnitude of $C_{i}$ is between 0.1 and $1 \mathrm{Fm}^{-2}$ ). In fact all the information relative to $C_{i}$ depends on the validity of the Gouy-Chapman theory in the domain of ionic concentrations and charges which are investigated.

Clearly, if we use $C_{G G C}$ instead of $C_{G C}$ in (4.1), then we may find a totally different behavior concerning $C_{i}$. For example, for high values of $\sigma$ we have a very high value of the Gouy-Chapman capacitance, as shown in the figures, then $C_{G C}^{-1}$ may be totally negligible compared to $C_{i}^{-1}$ and the experiment gives us $C_{\exp } \approx C_{i}$. When saturation is observed in our model then $C_{G G C} \ll C_{G C}$ and $C_{G G C}$ can contribute to $C_{\exp }$ while the contribution of $C_{G C}$ is negligible.

To illustrate another aspect of our results let us consider the traditional ideally polarizable electrode, but in which the diffuse layer is given by GGCM. This means that the experimental capacitance should be described by

$$
\frac{1}{C_{\exp }}=\frac{1}{C_{i}}+\frac{1}{C_{G G C}} \text {. }
$$

From the traditional approach, i.e. by using (4.1) we will introduce an effective inner layer capacitance, $C_{i}^{\text {eff }}$, defined according to

$$
\frac{1}{C_{i}^{e f f}}=\frac{1}{C_{e x p}}-\frac{1}{C_{G C}}=\frac{1}{C_{i}}+\frac{1}{C_{G G C}}-\frac{1}{C_{G C}} .
$$

For a given value of the charge, at extremely low ionic concentration there is no significant deviation between $C_{i}^{\text {eff }}$ and $C_{i}$. For moderate values of the concentration, and particularly for non-symmetrical ions, a large difference between $C_{i}^{\text {eff }}$ and $C_{i}$ appears. We will recover $C_{i}^{\text {eff }} \approx C_{i}$ at very high concentrations if $C_{G G C}$ and $C_{G C}$ are large enough compared to $C_{i}$. Thus, the traditional analysis may lead to erroneous conclusions if there are noticeable deviation from the Gouy-Chapman theory. Let us emphasize that the deviation investigated in this paper is a consequence of the bulk solution properties and cannot be attributed to any specific interfacial phenomena.

\section{Conclusions}

In addition to the hamiltonian which gives rise to the Non-Linear GouyChapman theory we have introduced some terms quadratic in the fields. They take into account the existence of short range correlations. The magnitude of these correlations is represented by three coupling constants $a_{i j}$, $i, j=+$ or - which contain all the specific behavior of the system i.e. the non-coulombic interaction between ions, the effect of the solvent and temperature. Just by changing the magnitude of these parameters we describe a variety of behaviors for the capacitance dependence on the charge density. Qualitative differences with the Gouy-Chapman theory appear both near the point of zero charge and for high values of the charge. At the pzc we may obtain a maximum or a finite slope instead of a minimum and at high charge densities we observe a saturation in the capacitance values instead of a continuous increase. The results are very sensitive to the asymmetry of ions which is expected in real systems. For asymmetric ions the capacitance extremum does not correspond to the pzc and a minimum at pzc is found only when the concentration approaches zero. It is interesting to note that 
this kind of behavior is generated by the bulk properties and not by any specific interfacial phenomena.

Our approach seems to be a good starting point for investigating a lot of phenomena occurring at charged interfaces or in the bulk of ionic solutions. At charged interfaces we may investigate more sophisticated hamiltonians which are able to describe specific interfacial phenomena as, for instance, the existence of interfacial phase transitions. Some results in this field are being prepared. In parallel, the formalism can be useful for studying the criticality in ionic solutions, some progress in this direction is expected.

\section{Acknowledgements}

This paper results from the cooperation of our laboratories carried out within the framework of the agreement between CNRS in France and the Polish Academy of Sciences.

\section{References}

[1] Hansen J.P. McDonald I.R., Theory of Simple Liquids, Academic Press, 1976.

[2] Sullivan D.E., Telo da Gama M.M., Wetting Transition and Multilayer Adsorption at Fluid Interfaces. In: Fluid Interfacial Phenomena, ed. by Croxton C.A., Wiley, 1986.

[3] Amokrane S., Badiali J.P., Analysis of the Capacitance of the Metal Solution Interface. In: "Modern Aspects of Electrochemistry", Vol. 22, ed. by J.O'M. Bockris, B.E.Conway and R.E.White, Plenum, 1992, p. 1-192.

[4] Schmickler W., Henderson D. New Models for the Structure of the Electrochemical Interface. // Prog. Surf. Sci., 1986, vol. 22 No. 5, p. 323-419.

[5] Stafiej J., Borkowska Z., Badiali J. P. A descrption of electrified interfaces based on methods of statistical field theory // J. Electroanal. Chem, 1995, vol. 395, p. 1-14.

[6] Stafiej J., Dymitrowska M., Badiali J. P. Field theoretic aproach of electrified interfaces. // Electrochim. Acta, 1996, vol. 41, p. 2107-2113.

[7] Stafiej J., Badiali J. P. On a new theoretical approach to electrified interfaces. // J. Electroanal. Chem., 1996, vol. 409, p. 73-78.

[8] Stafiej J., Ekoka A., Borkowska Z., Badiali J. P. New theoretical description of electrified interfaces.// J. Chem. Soc., Faraday Trans., 1996, vol. 92, p. $3677-3682$.

[9] Carnie S.L., Torrie G.M. The statistical mechanics of the electrical double layer. // Adv. Chem. Phys., 1984, vol. 56, p. 141-253.

[10] Grahame D.C. Thermodynamic properties of the electrical double layer. // Chem. Revs, 1947, vol. 41, p. 441-443.

[11] Delahay P. Double layer and electrode kinetics. New York, Interscience, 1965, p. $1-112$.

[12] Nabutovskii V. M., Nemov N.A., Peisakhovich Yu.G. Correlation functions for the electrolyte near the critical point of pure solvent. // Mol. Phys., 1985, vol. 54, p. 979-987.

[13] Hoye J.S., Stell G. Effect of solute on the solvent critical behavior 1. General formalism and the possibility of charge-density waves. // J. Phys. Chem., 1990, vol. 94, p. 7899-7907.

[14] Badiali J.P. Structure of a polar fluid near a wall. Exact asymptotic behavior of the profile, relation with the electrostriction phenomena and the Kerr effect. // J. Chem. Phys., 1989, vol. 90, No. 9, p. 4401-4412.

[15] Fawcett W. R. In: Frontiers in Electrochemistry, ed. by J. Lipkowski and P.N. Ross, Weinheim, in press. 


\title{
СПРОЩЕНИЙ ОПИС СТРУКТУРИ ІОННИХ РОЗЧИНІВ
} БІЛЯ ЗАРЯДЖЕНИХ ПОВЕРХОНЬ

\author{
Й.Стаф'є, А.Екока, З.Борковська, Ж.-П.Бадьялі
}

\begin{abstract}
У цій статті теоретикопольовий підхід використано для опису властивостей іонних розчинів поблизу зарядженої стінки. Відмічаються відмінності від теорії Гуі-Чепмена поблизу точки нульового заряду та для високих значень заряду. В точці нульового заряду отримано максимум замість мінімуму, а при високих значеннях густини заряду спостерігається насичення для величини ємності замість їі неперервного зростання. Результати є дуже чутливі до асиметрії іонів, що припускається в реальних системах. Наш підхід може бути доброю стартовою точкою для дослідження різних явищ на заряджених поверхнях і для об'ємних властивостей іонних розчинів.
\end{abstract}

